\title{
Deficiencia de vitamina $D$ en madres y neonatos mexicanos
}

\author{
Erika del Carmen Ochoa-Correa, Pedro Alberto García-Hernández, Jesús Zacarías Villarreal-Pérez, \\ Consuelo Treviño-Garza, Isaías Rodríguez-Balderrama, Laura Elia Martínez-de Villarreal, \\ Carlos Alberto Zapata-Castilleja y Manuel Enrique De la O-Cavazos \\ Departamento de Pediatría, Hospital Universitario Dr. José Eleuterio González, Universidad Autónoma de Nuevo León, Monterrey, N.L., México
}

\section{Resumen}

Objetivo: Establecer la prevalencia de la deficiencia de vitamina $D$ en madres y sus neonatos, y analizar factores de riesgo asociados. Métodos: Estudio observacional, transversal y prospectivo. Se incluyeron 191 puérperas y sus neonatos de término. Se analizaron las concentraciones séricas de 25 hidroxi-vitamina $D$ (inmunoensayo enzimático) y se correlacionaron con factores de riesgo maternos y datos antropométricos neonatales. Resultados: Se presentó deficiencia en el $61 \%$ de las madres, y en el $26 \%$ insuficiencia. El 98\% de los neonatos mostraron deficiencia (66\% grave). Existe una correlación positiva entre las concentraciones de vitamina $D$ de madres y neonatos ( $r 2=0.173 ; p=0.017)$. Los valores más bajos se presentaron en otoño (15.75 y $6 \mathrm{ng} / \mathrm{ml}$ materna y neonatal, respectivamente). No hubo correlación entre los valores de vitamina $D$ materna y su alimentación, fototipo, tiempo de exposición solar o índice de masa corporal. Conclusiones: Este es el primer estudio que muestra la existencia de una alta deficiencia de vitamina $D$ en madres y sus neonatos mexicanos.

PALABRAS CLAVE: Deficiencia de vitamina D. Neonatos. Embarazo. Vitamina D.

\begin{abstract}
Objective: The purpose of this study is to establish the prevalence of vitamin $D$ deficiency and their newborns and analyze the risk factors related to this deficiency. Methods: This is an observational, transversal, and prospective study. It included 191 puerperal women and their full-term newborns. Serum 25 hydroxyvitamin $D$ values were analyzes by enzyme immunoassay. Results: $61 \%$ of the puerperal presented deficiency and $26 \%$ insufficiency of vitamin $D$. In the newborn group $98 \%$ showed deficiency and $66 \%$ of them presented severe deficiency. There is a positive correlation between the values of vitamin $D$ in mothers and their newborns $\left(r^{2}=0.173 \mathrm{ng} / \mathrm{ml} ; p=0.017\right)$. The lowest levels were in autumn. $(15.75 \mathrm{ng} / \mathrm{mL} \mathrm{mothers}, 6 \mathrm{ng} / \mathrm{mL}$ newborns). There was no correlation between vitamin $D$ levels in mothers and their dietary intake, maternal skin type, sun time exposure and prenatal body mass index. Conclusions: This is the first study that shows the existence of a high deficiency of vitamin $D$ in Mexican mothers and their newborns.
\end{abstract}

KEY WORDS: Vitamin D deficiency. Newborns. Pregnancy. Vitamin D.

\author{
Correspondencia: \\ M.E. De la O-Cavazos \\ Av. Francisco I. Madero y Gonzalitos, s/n \\ Col. Mitras Centro \\ C.P. 64460 , Monterrey, N.L., México \\ E-mail: delaocavazos@yahoo.com
}

Fecha de recepción: 17-01-2017

Fecha de aceptación: 28-04-2017

DOI:10.24875/GMM.17002980
Gac Med Mex. 2017;153:559-565

Contents available at PubMed www.gacetamedicademexico.com 


\section{Introducción}

La deficiencia de vitamina $\mathrm{D}$ se considera un problema de salud pública en todo el mundo. La relevancia de esta condición estriba en que, además de su función clásica en la homeostasis ósea, la vitamina $D$ también interviene en diferentes procesos biológicos importantes para el crecimiento y el desarrollo tanto en la vida prenatal como en la posnatal ${ }^{1-4}$. Existen factores de riesgo que se han asociado a esta deficiencia, como residir en latitudes del Norte, una limitada exposición solar, el uso regular de bloqueadores solares, piel oscura, obesidad y síndromes de malabsorción ${ }^{1}$. En el embarazo, la deficiencia de vitamina $D$ se asocia a complicaciones como la preeclampsia; en el neonato, a bajo peso e hipocalcemia, así como a pobre crecimiento posnatal, fragilidad ósea y aumento en la incidencia de enfermedades autoinmunes ${ }^{1,3,4}$.

Existen reportes sobre la deficiencia de vitamina $D$ en madres y sus neonatos en diversas poblaciones, la cual es muy variable incluso en el mismo país. En los EE.UU., Mulligan, et al. ${ }^{1}$ reportan una prevalencia de esta deficiencia del $5-50 \%$ en las madres y del $54 \%$ en los neonatos; por su parte, Johnson, et al. ${ }^{5}$ mencionan que el $30 \%$ de las mujeres embarazadas hispanas tuvieron deficiencia de vitamina D. En la India, Jain, et al. ${ }^{6}$ encontraron deficiencia de vitamina $\mathrm{D}$ en el $81 \%$ de las mujeres en el embarazo y en el $66.7 \%$ en los neonatos. Por otro lado, en países como Grecia, Bélgica y Holanda, el $10-30 \%$ de las mujeres presentan deficiencia de vitamina $D$ en el embarazo ${ }^{1,5}$.

Debido al riesgo de desarrollar problemas tanto maternos como fetales ante una condición de deficiencia de vitamina $D$, diversos países han implementado protocolos de suplementación durante el embarazo y en el período neonatal ${ }^{7-9}$. A la fecha, en México no existen reportes sobre las concentraciones séricas de 25-hidroxi-vitamina $\mathrm{D}(25 \mathrm{OHD})$ en neonatos y sus madres que pudieran fundamentar una política de suplementación. El objetivo de esta investigación es conocer los valores de vitamina $\mathrm{D}$ en esta población y correlacionarlos con los diversos factores de riesgo que pueden condicionar su deficiencia.

\section{Métodos}

Se llevó a cabo un estudio observacional, transversal y prospectivo en puérperas y sus neonatos atendidos en el Hospital Universitario Dr. José Eleuterio González, de Monterrey, N.L., México. La población de estudio es residente de Monterrey, ciudad localizada en la latitud Norte de 25'40'11', en el periodo del 20 de mayo de 2013 al 30 de septiembre de 2014. El protocolo fue autorizado por los Comités de Investigación y de Ética de la institución (PE13-013). El consentimiento informado de los menores fue firmado por ambos padres.

Los criterios de inclusión fueron: madres mexicanas, de cualquier edad, con embarazo a término; y neonatos de término (37-41 semanas de gestación) y sanos (sin complicaciones durante el nacimiento). Los criterios de exclusión fueron gestación múltiple y neonatos con malformaciones mayores. Los criterios de eliminación fueron datos incompletos o muestra insuficiente del binomio madre-hijo.

Además de la historia clínica completa y documentada en el expediente clínico, a todas las madres se les aplicó un cuestionario para la obtención de datos antropométricos (peso prenatal y peso al momento del ingreso a la atención del nacimiento), uso de medicamentos y enfermedades. Se aplicó un cuestionario de frecuencia de alimentos de vitamina $D^{10} y$ consumo de suplementos de vitamina $D$, colecalciferol y ergocalciferol (diarios y semanales).

Se evaluó el color de piel materna, clasificando el fototipo de acuerdo con Fitzpatrick ${ }^{11}$, así como el tiempo de exposición solar en minutos/día.

En los neonatos se obtuvo el registro de fecha de nacimiento, con la finalidad de determinar la estación del año imperante en el momento del nacimiento, tomando en cuenta lo siguiente primavera los meses de marzo a mayo, verano de junio a agosto, otoño de septiembre a noviembre, e invierno de diciembre a febrero; edad gestacional, la cual se estableció por el método de Capurro; sexo; Apgar a 1 y 5 minutos; peso en gramos; talla y perímetro cefálico en centímetros, lo cual se realizó mediante el uso de un tallímetro (SECA 210, Alemania), una cinta métrica (SECA 201,Alemania) y una báscula digital (SECA 354, Alemania). Las variables antropométricas se analizaron con z score y los percentiles por medio de la gráfica de Fenton.

Para la cuantificación de vitamina D3, en las madres se obtuvieron 5-7 $\mathrm{ml}$ de sangre venosa periférica de la región antecubital, en un lapso de entre 24 y 48 horas posteriores al evento obstétrico. En el recién nacido se obtuvo una muestra de 1-2 ml de sangre capilar, por punción (al momento de tomar el tamiz metabólico neonatal), a las 24-48 horas de vida extrauterina. Una vez colectadas las muestras, el suero se centrifugó por 20 minutos y se guardaron en alícuotas a una temperatura de $-20{ }^{\circ} \mathrm{C}$. Todas las muestras fueron procesadas al mismo tiempo por el método de inmunoensayo enzimático (ELISA), en un equipo Cobas 6000 Hitachi 
(Roche, Suiza). Para ello se utilizó el kit de ELISA para vitamina D3 Cloud-Clone Corp. ${ }^{\odot}$, Test CEA920Ge 96, Houston, Tx, EE.UU., cuyo rango de detección es de 4.94-400 ng/ml, siguiendo las especificaciones del proveedor. Dicho instrumento cuenta con una sensibilidad de medición mínima de $2.15 \mathrm{ng} / \mathrm{ml}$, una alta especificidad para detectar vitamina D3 y una precisión intraensayo $\mathrm{CV}<10 \%$ e interensayo $\mathrm{CV}<12 \%$.

Las concentraciones séricas de $250 \mathrm{HD}$ para el binomio madre-hijo se dividieron utilizando la clasificación de la Sociedad de Endocrinología Clínica ${ }^{12}$ normal entre 30 y $100 \mathrm{ng} / \mathrm{ml}$, insuficiencia con un rango de 20 a $29 \mathrm{ng} / \mathrm{ml}$, deficiencia entre 12 y $19 \mathrm{ng} / \mathrm{ml}$, y deficiencia grave $<11 \mathrm{ng} / \mathrm{ml}$.

Para el análisis estadístico se usó el paquete SPSS 20 (IBM, Armonk, NY, EE.UU.), obteniendo medidas de tendencia central y dispersión, frecuencias y porcentajes. Se consideró estadísticamente significativo un valor de $p<0.05$, y se utilizó la prueba $t$ de Student para muestras independientes. Para confirmar la distribución normal se usó la prueba de Kolmogorov-Smirnov, y para las variables categóricas se utilizó la prueba de ji al cuadrado.

Adicionalmente, se realizó una prueba de correlación lineal de Pearson univariada entre los valores de vitamina $\mathrm{D}$ materno y neonatal y la variable de peso neonatal. Finalmente se utilizó un modelo multivariado de regresión lineal, con los valores de $250 \mathrm{HD}$ maternos como variable dependiente, y con el consumo de vitamina $D$ en la dieta materna, el fototipo de piel, el tiempo de exposición solar y el índice de masa corporal (IMC) al inicio de la gestación como covariables.

\section{Resultados}

Se reclutaron 194 binomios madre-hijo y se excluyeron tres por muestra sanguínea insuficiente de los neonatos, quedando un total de 191 binomios.

El $64 \%$ de las madres eran adultas y el $36 \%$ eran adolescentes; la mayoría se dedicaba al hogar, tenían escolaridad básica y una pareja estable. Al analizar las variables antropométricas maternas, se observó que la tercera parte de las participantes iniciaron el embarazo con sobrepeso u obesidad, y al término del mismo este número aumentó al 78\% (Tabla 1). Por su parte, las medidas antropométricas neonatales se encontraron dentro de los percentiles normales (Tabla 2).

La media de $250 H D$ fue de $19.74 \pm 10.21 \mathrm{ng} / \mathrm{ml}$ en las madres y de $9.42 \pm 3.53 \mathrm{ng} / \mathrm{ml}$ en los neonatos. Esto evidencia que solo el $13 \%$ de las madres presentaron valores normales (>32 $\mathrm{ng} / \mathrm{ml}$ ), mientras que el $26 \%$ mostró insuficiencia $(20-31 \mathrm{ng} / \mathrm{ml})$ y el $61 \%$ tuvo deficiencia $(<20 \mathrm{ng} / \mathrm{ml})$. Más relevante aún
Tabla 1. Variables demográficas y somatometría de las madres, donde para el IMC al final de la gestación se tomó en cuenta el peso registrado al momento del ingreso

\begin{tabular}{|c|c|}
\hline & Total $n=191(\%)$ \\
\hline \multicolumn{2}{|l|}{ Edad } \\
\hline Adolescentes ( $\leq 19$ años) & $69(36)$ \\
\hline Adultas ( $\geq 20$ años) & $122(64)$ \\
\hline Ocupación & $169(88)$ \\
\hline Hogar & $9(5)$ \\
\hline Estudiante & $13(7)$ \\
\hline \multicolumn{2}{|l|}{ Empleada } \\
\hline Estado civil & $32(17)$ \\
\hline Soltera & $48(25)$ \\
\hline Casada & $104(55)$ \\
\hline Unión libre & $2(1)$ \\
\hline \multicolumn{2}{|l|}{ Divorciada } \\
\hline Escolaridad & $2(1)$ \\
\hline Analfabeta & $34(18)$ \\
\hline Primaria & $87(46)$ \\
\hline Secundaria & $61(32)$ \\
\hline Preparatoria & $4(2)$ \\
\hline \multicolumn{2}{|l|}{ Licenciatura } \\
\hline Peso inicio embarazo (kg) & $60,39 \pm 13,44$ \\
\hline IMC prenatal & $24,31 \pm 5,08$ \\
\hline Normal $(<25)$ & $122(64)$ \\
\hline Sobrepeso (25-29.99) & $40(21)$ \\
\hline Obesidad (> 30) & $29(15)$ \\
\hline Peso final del embarazo (kg) & $72,84 \pm 13,87$ \\
\hline IMC final del embarazo & $29,35 \pm 5,34$ \\
\hline Normal $(<25)$ & $42(22)$ \\
\hline Sobrepeso (25-29.99) & $76(40)$ \\
\hline Obesidad (> 30) & $73(38)$ \\
\hline \multicolumn{2}{|l|}{ Gestas previas } \\
\hline 0 & $74(39)$ \\
\hline 1 & $59(31)$ \\
\hline 2 & $33(17)$ \\
\hline $3+$ & $25(13)$ \\
\hline
\end{tabular}

IMC: índice de masa corporal.

resulta que todos los neonatos presentaron valores de $250 \mathrm{HD}$ anormalmente bajos: $2 \%$ de insuficiencia y $98 \%$ de deficiencia. De estos últimos, el $66 \%$ tenían deficiencia grave, definida por valores $<11 \mathrm{ng} / \mathrm{ml}$.

Por otro lado, existe una débil correlación positiva entre los valores de $250 \mathrm{HD}$ de la madre y las concentraciones séricas de $250 \mathrm{HD}$ de los neonatos $\left(r^{2}=0.173 ; p=0.017\right)$. Asimismo, las concentraciones séricas de $250 \mathrm{HD}$ maternas predicen los valores de la 25OHD neonatal $\left(r^{2}=0.030 ; p=0.017\right)$.

Siguiendo la clasificación utilizada con anterioridad, donde se dividió la muestra en grupos de madres adolescentes y madres adultas, no se encontraron diferencias estadísticamente significativas en los valores séricos de 250HD entre ambos grupos, así como tampoco en los neonatos (Tabla 3). 
El promedio de tiempo diario de exposición solar de las madres durante el embarazo fue de $85 \pm 91.06$ minutos (rango: 5-600). El consumo diario de vitamina $D$ a través de la dieta fue en promedio de $172 \pm 76.31 \mathrm{UI}$ (rango: 14-341). Únicamente 52 (27.22\%) madres recibieron suplemento de vitamina $D$, con una media de $291 \pm 76.44$ UI/día (rango: 171-500) (Tabla 4); sin embargo, no se encontraron diferencias entre las madres que consumieron suplementos y las que no los habían consumido.

El fototipo cutáneo materno predominante fue el $3(40.31 \%)$, seguido de los fototipos 4,5 y 2 . No se presentaron fototipos 1 y 6 . Al comparar las medias de 25OHD de acuerdo con el fototipo, no se encontró diferencia estadísticamente significativa $(p=0.62)$

En la figura 1 se muestran las medias de los valores séricos de 250HD de la madre y del recién nacido, por estación del año al momento del nacimiento. Los valores más bajos se encontraron en otoño.

En la correlación multivariada no se halló asociación de los valores de 250HD materna con el consumo de vitamina $D$ en la dieta $\left(r^{2}=-0.10 ; p=0.79\right)$ ni con el fototipo cutáneo materno $\left(r^{2}=-0.019 ; p=0.79\right)$, el tiempo de exposición solar $\left(r^{2}=-0.44 ; p=0.54\right)$, el IMC al final del embarazo $\left(r^{2}=-0.124 ; p=0.08\right) \circ$ la estación del año $\left(r^{2}=0.033 ; p=0.65\right)$.

\section{Discusión}

La vitamina $D$ es la principal hormona en la regulación del metabolismo fosfocálcico. Su deficiencia es un problema de salud en todo el mundo ${ }^{1-3,7,13}$ y hay

\section{Tabla 2. Variables neonatales}

\begin{tabular}{lc}
\hline & Total $\mathbf{n}=\mathbf{1 9 1}(\%)$ \\
\hline Vía nacimiento & $113(59)$ \\
Parto & $78(41)$ \\
Cesárea & \\
Sexo & $95(49)$ \\
Masculino & $96(51)$ \\
Femenino & Media $(\mathrm{DE})$ \\
Apgar & $8( \pm 0,60)$ \\
1 minuto & $9( \pm 0,80)$ \\
5 minutos & $39,1( \pm 1,19)$ \\
Edad gestacional & $3254,71( \pm 411,32)$ \\
Peso (g) & $-0,15( \pm 0,85)$ \\
Z score & $50,33( \pm 1,81)$ \\
Talla (cm) & $34,30( \pm 1,50)$ \\
Perímetro cefálico (cm) &
\end{tabular}

enfermedades en la edad pediátrica que cursan con alteraciones en el metabolismo de la vitamina $D$, como raquitismo, insuficiencia renal crónica, enfermedades alérgicas, cáncer, patologías que se asocian con malabsorción de grasas (fibrosis quística, enfermedad celíaca), obesidad y sobrepeso ${ }^{7,12,14}$. Actualmente, en todos los continentes, incluyendo países iberoamericanos como España y Argentina, se han descrito poblaciones con alta deficiencia de esta vitamina ${ }^{15,16}$.

Específicamente en los recién nacidos, la reserva de vitamina $D$ depende de la transferencia materna a través de la vía placentaria. A partir del nacimiento, este suministro corre por cuenta de su propia producción y del aporte exógeno'. En el embarazo se asocia con preeclampsia grave y en el neonato con hipocalcemia, raquitismo (principalmente en prematuros) $)^{14}$ y peso bajo para la edad gestacional'1. Los valores óptimos de vitamina $D$ en el embarazo y en la etapa neonatal aún se mantienen en controversia. Existen la clasificación del Instituto de Medicina y la clasificación de la Sociedad de Endocrinología Clínica' ${ }^{2}$, siendo esta última la empleada en el presente trabajo, la cual establece como valores de normalidad entre 32 y $100 \mathrm{ng} / \mathrm{ml}$, como insuficiencia un rango de 20 a $31 \mathrm{ng} / \mathrm{ml}$, como deficiencia entre 12 y $19 \mathrm{ng} / \mathrm{ml}$, y como deficiencia grave $<11 \mathrm{ng} /$ $\mathrm{ml}$. Esta clasificación es la más utilizada actualmente y ha mostrado que los valores de insuficiencia se asocian con alteraciones extraesqueléticas.

En este reporte se evidenció que el $61 \%$ de las madres y el $98 \%$ de los neonatos presentaron valores de deficiencia de 25OHD. Dichos resultados coinciden con investigaciones internacionales en las que se confirma que la deficiencia de vitamina $D$ tiene una alta prevalencia entre las mujeres embarazadas y sus neonatos ${ }^{16-20}$. Por otro lado, se observó una correlación débilmente positiva entre los valores de 25OHD materna y neonatal $\left(r^{2}=0.173 ; p=0.017\right)$. Esta correlación fue similar a la reportada por Abbasian, et al..$^{13}$ para los valores de $25 \mathrm{OHD}$ de suero materno y sangre de cordón en una población iraní $\left(r^{2}=0.12 ; p=0.053\right)$.

En nuestro país existen reportes en relación con los valores sanguíneos de vitamina $D$ en diferentes grupos de edad. En la Encuesta Nacional de Salud ${ }^{21}$ se reportó que el $9.8 \%$ de los adultos presentaron deficiencia de $250 \mathrm{HD}$, el $20 \%$ insuficiencia y aproximadamente el $70 \%$ suficiencia, mientras que Elizondo, et al. ${ }^{22}$ analizaron pacientes pediátricos de 6-12 años, obesos y de la misma región geográfica que nuestro estudio, y encontraron que presentaban deficiencia en una proporción similar a la hallada en las madres en el presente trabajo. No se encontraron reportes en los que se hayan analizado los 


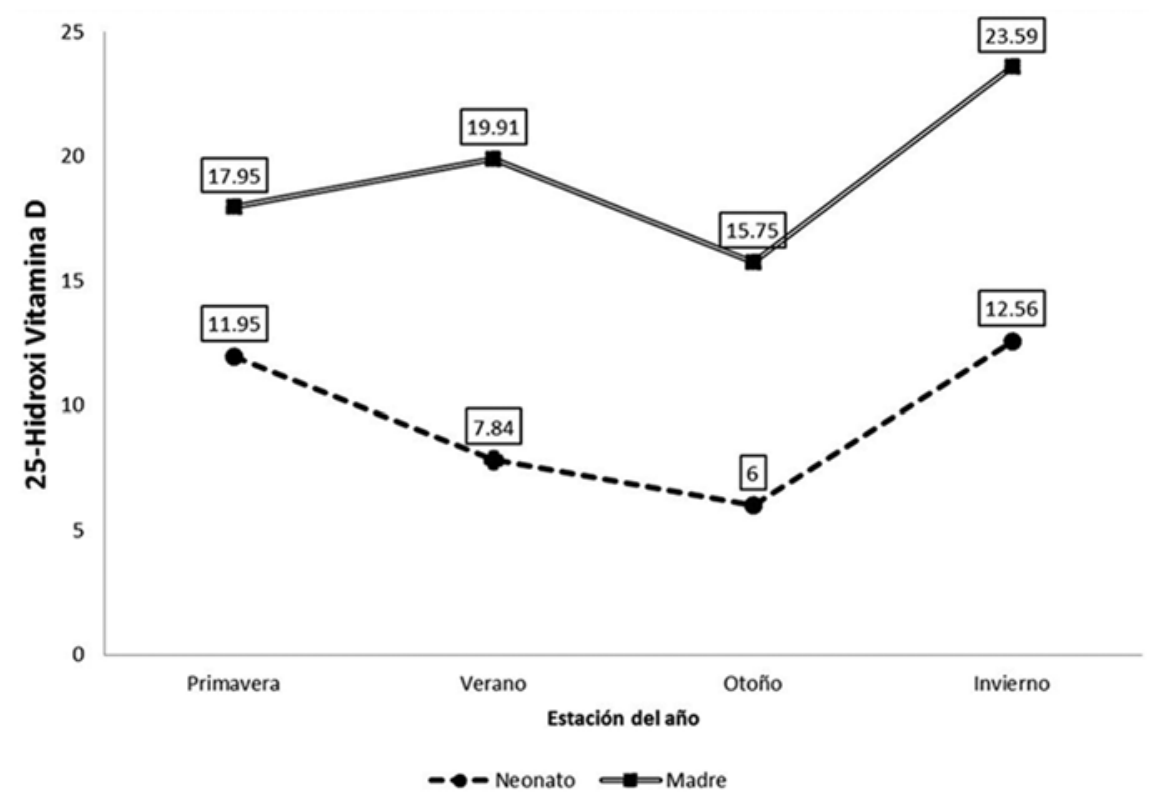

Figura 1. Valores promedio de 25-hidroxivitamina D en madres y neonatos según la estación del año. Se evidencia la variabilidad estacional, encontrando los valores más bajos en otoño.

Tabla 3. Comparación de la 25 hidroxi-vitamina D sérica materna y neonatal de acuerdo con la edad de la madre

\begin{tabular}{|c|c|c|c|c|}
\hline Clasificación & Total $n=191(\%)$ & Madres adolescentes $\mathrm{n}=69(\%)$ & Madres adultas $n=122(\%)$ & p \\
\hline \multicolumn{5}{|l|}{ Materna } \\
\hline Normal (32-100 ng/ml) & $26(13)$ & $9(13)$ & $17(14)$ & 0.98 \\
\hline Insuficiencia (20-31 ng/ml) & $49(26)$ & $18(26)$ & $31(25)$ & \\
\hline Deficiencia (12-19 ng/ml) & $95(50)$ & $35(51)$ & $60(49)$ & \\
\hline $\begin{array}{l}\text { Deficiencia grave } \\
<11 \mathrm{ng} / \mathrm{ml})\end{array}$ & $21(11)$ & $7(10)$ & $14(12)$ & \\
\hline \multicolumn{5}{|l|}{ Neonatal } \\
\hline Normal (32-100 ng/ml) & 0 & 0 & 0 & 0.3 \\
\hline Insuficiencia (20-31 ng/ml) & $3(2)$ & $1(1)$ & $2(2)$ & \\
\hline Deficiencia (12-19 ng/ml) & $62(32)$ & $23(33)$ & $39(32)$ & \\
\hline $\begin{array}{l}\text { Deficiencia grave } \\
(<11 \mathrm{ng} / \mathrm{ml})\end{array}$ & $126(66)$ & $45(65)$ & $81(66)$ & \\
\hline
\end{tabular}

Tabla 4. Consumo de vitamina D por dieta y suplemento

\begin{tabular}{lccc}
\hline Consumo por vía oral & $\begin{array}{c}\text { Total } \\
\mathbf{n = 1 9 1}\end{array}$ & Media $\pm \mathbf{D E}$ & Rango \\
\hline Dieta (UI/día) & 191 & $172 \pm 76,31$ & $14-341$ \\
Suplemento (UI/día) & 52 & $291 \pm 76,44$ & $171-500$ \\
Dieta+suplemento (UI/día) & 52 & $462 \pm 105,49$ & $239-676$ \\
\hline DE: desviación estándar. & & &
\end{tabular}

valores de vitamina $\mathrm{D}$ en el binomio madre-hijo, además de ser este el primer estudio en neonatos mexicanos.

En la presente investigación no se encontraron diferencias entre los valores sanguíneos de las madres adolescentes y de las madres adultas. Lo anterior difiere de lo reportado en la literatura en otros grupos poblacionales, en donde las madres adolescentes tienen una mayor pérdida de masa ósea desde el comienzo del embarazo hasta la sexta semana posparto, indicando que la prevalencia de deficiencia de vitamina $D$ en ellas es mayor ${ }^{13,23-25}$.

En nuestro estudio se documentó que la tercera parte de las madres presentaron sobrepeso $u$ obesidad al inicio del embarazo, lo cual se incrementó significativamente al final del mismo. Aunque no se halló correlación entre el IMC materno al final del embarazo y los valores de $250 H D$, se ha reportado que la deficiencia de vitamina $D$ puede relacionarse con obesidad, para lo cual se han propuesto dos mecanismos: 1) que la grasa corporal excesiva secuestra la vitamina D circulante, 2) que la deficiencia de vitamina $D$ aumenta el acúmulo de grasa en el organismo ${ }^{26-28}$, y 3) la asociación de sobrepeso/obesidad y resistencia a la insulina ${ }^{29}$. Elizondo, et al. ${ }^{22}$ evidenciaron que existe mayor prevalencia de 
deficiencia de 25OHD en el grupo de obesos, comparado con un grupo control de no obesos, pero en su estudio no se encontró una correlación significativa entre los niveles de $25 \mathrm{OHD}$ y el IMC de las madres al final del embarazo. Una debilidad de este estudio es que no fueron analizados los valores de 250HD al inicio del embarazo, motivo por el cual no se logró realizar su correlación con el IMC al inicio del mismo.

Es importante considerar el fototipo de piel, ya que la concentración de melanina regula la cantidad de radiación UVB que penetra hasta alcanzar las capas epidérmicas, con la máxima concentración de 7-dehidrocolesterol ${ }^{30,31}$. El fototipo cutáneo, definido como la capacidad de adaptación al sol que tiene cada persona, queda clasificado en seis categorías según Fitzpatrick, que van desde el fototipo 1 , correspondiente a individuos con piel blanca y pelo rojo, hasta el fototipo 6 para personas con piel oscura y pelo negro ${ }^{11,32}$.

El fototipo cutáneo materno predominante fue el 3 . Al comparar las medias por fototipos, no se observó una diferencia estadísticamente significativa. Estos resultados son diferentes a lo reportado en la literatura internacional, donde se asevera que los valores de vitamina D están asociados inversamente con el fototipo cutáneo de la madre, de tal forma que cuanto mayor es este, mayor riesgo de hipovitaminosis puede existir ${ }^{11,32-34}$.

Otro de los aspectos a destacar es que, a pesar de que la latitud en la que se encuentra la ciudad de Monterrey $\left(25^{\circ} 40^{\prime} 11^{\prime \prime} \mathrm{N}\right)$ cuenta con abundante radiación solar, no se detectó una diferencia estadísticamente significativa en la comparación de las medias de los valores de 25OHD por tiempo de exposición solar, aunque sí encontramos una variación muy importante entre las participantes. Estos hallazgos coinciden con los de otras poblaciones que presentan una exposición solar alta, en latitudes similares a la nuestra. Tal es el caso de la región del Mediterráneo, India y Túnez $z^{6,35,36}$.

Al analizar los valores de 250HD y la estación del año al momento del evento obstétrico no se encontró ninguna correlación; incluso, los valores más bajos fueron en otoño, diferente a lo reportado en la literatura, donde los valores más bajos de vitamina $D$ aparecen en las estaciones de invierno y el comienzo de la primavera ${ }^{6,33}$. Una hipótesis sería que la región geográfica donde se desarrolló el estudio, aunque predomina un clima seco y semiseco, presenta una precipitación media de $650 \mathrm{~mm}$ anuales y las lluvias se presentan en los meses de agosto y septiembre ${ }^{37}$; por lo tanto, disminuye la exposición solar en estos meses, y como la síntesis de vitamina $D$ se desarrolla en aproximadamente 3 semanas, esto puede explicar que se detectaran los valores más bajos de 250HD a principios de esta estación del año.
El principal aporte exógeno de la vitamina $\mathrm{D}$ es dado por el ergocalciferol $\left(D_{2}\right)$ a través de la dieta y el colecalciferol $\left(\mathrm{D}_{3}\right)^{38}$. Se tomó en cuenta el consumo diario de vitamina $\mathrm{D}$ en la dieta de las participantes del estudio. Este resultó bajo, similar a lo reportado por Ortigosa, et al. ${ }^{39}$ en una muestra que incluye mujeres sudamericanas radicadas en Barcelona, España. Dicho resultado puede ser secundario al hecho de que sean originarias de Sudamérica y continúen con hábitos y costumbres alimenticios similares a los de su lugar de origen.

Finalmente, se evidenció que solo el $27 \%$ de las madres consumieron suplementos de vitamina $D$ durante la gestación. Esto mediante dosis en un rango de 171 a 500 Ul/día, cantidades mucho más bajas que las recomendadas internacionalmente de 600-4000 Ul/día. ${ }^{40}$ Ello se debe a que en México no hay una política para la suplementación de vitamina $D$ en el embarazo, motivo por el cual aún no se regula la cantidad que de esta deben tener los multivitamínicos que se prescriben durante el embarazo. En México se considera la deficiencia de vitamina D como una patología rara ${ }^{14}$, sobre todo por la falta de evidencia científica, ya que existen muy pocos estudios de prevalencia y actualmente ninguno incluye a puérperas y sus neonatos. Los resultados obtenidos en la presente investigación sustentan que existe una alta prevalencia de deficiencia de vitamina $\mathrm{D}$ al nacimiento, aunque no se logró evidenciar complicaciones inmediatas en el neonato. Sin embargo, esto no excluye la posibilidad de tener a largo plazo complicaciones relacionadas con la hipovitaminosis D. Está demostrado que la leche materna tiene cantidades muy bajas de vitamina $\mathrm{D}$ (25-78 UI/I), y un lactante alimentado al seno materno exclusivo tiene una mayor predisposición a presentar deficiencia de vitamina $D^{41}$. Por lo tanto, se sugiere que deba considerarse como un problema de salud pública y realizar acciones de suplementación, tanto en el embarazo como en la etapa neonatal, aumentado las dosis recomendadas por la Norma Oficial Mexicana NOM-051SCFI/SSA1-2010, que sugiere una ingesta diaria de 224 UI, basada en las recomendaciones del Instituto Nacional de Ciencias Médicas y Nutrición Salvador Zubirán ${ }^{14}$.

Se recomienda realizar estudios prospectivos en México con diferentes dosis de suplementación en los neonatos y lactantes entre 400 y $1000 \mathrm{UI}$ diarias, incluyendo como variables los diferentes tipos de alimentación, para establecer la dosis óptima en este grupo de población.

\section{Conclusiones}

Existe una alta prevalencia de deficiencia de vitamina $D$ en las madres y sus neonatos, independientemente 
de factores como la edad materna, el peso, el tiempo de exposición solar, la latitud de la ciudad donde se radica, la estación del año y el fototipo de piel.

Para futuras investigaciones sería conveniente hacer un estudio poblacional más extenso, analizar los polimorfismos del gen receptor de la vitamina D más frecuentes y utilizar diferentes esquemas de suplementación de vitamina $D$.

\section{Recursos financieros}

Este estudio fue realizado con recursos propios del Departamento de Pediatría y del Servicio de Endocrinología del Hospital Universitario Dr. José Eleuterio González, de la Universidad Autónoma de Nuevo León. Los autores declaran que no existen fuentes de financiación externa que declarar.

\section{Agradecimientos}

A la Q.C.B. Laura Jaqueline Cortés Gallardo, M.A. Gloria Alejandra Jasso de la Peña, Dra. Brenda Leticia Méndez Guerrero y Dr. Neri Alejandro Álvarez Villalobos por su valiosa colaboración.

\section{Bibliografía}

1. Mulligan ML, Felton SK, Riek AE, et al. Implications of vitamin D deficiency in pregnancy and lactation. Am J Obstet Gynecol. 2010;202:429. e1-9.

2. Bischoff-Ferrari H. Vitamin D - Role in pregnancy and early childhood. Ann Nutr Metab. 2011;59:17-21.

3. Holik MF. Vitamin D deficiency. N Engl J Med. 2007;357:266-81.

4. Bodnar LM, Catov JM, Simhan HN, et al. Maternal vitamin D deficiency increases the risk of preeclampsia. $J$ Clin Endocrinol Metab. 2007;92:3517-22.

5. Johnson DD, Wagner CL, Hulsey TC, et al. Vitamin D deficiency and insufficiency is common during pregnancy. Am J Perinatol. 2011; 28:7-12.

6. Jain V, Gupta N, Kalaivani M, et al. Vitamin D deficiency in healthy breastfed term infants at 3 months and their mothers in India seasonal variation \& determinants. Indian J Med Res. 2011;133:267-73.

7. Saggese G, Vierucci F, Boot AM, et al. Vitamin D in childhood and adolescence: an expert position statement. Eur $\mathrm{J}$ Pediatr. 2015;174:565-76.

8. Canadian Paediatric Society. Vitamin D supplementation: recommendations for Canadian mothers and infants. Paediatr Child Health. 2007; 12:583-9.

9. American College of Obstetricians and Gynecologists. Vitamin D screening and supplementation during pregnancy. Committee Opinion No. 495. Obstet Gynecol. 2011;118:197-8.

10. Taylor C, Lamparello B, Kruczek K, et al. Validation of a food frequency questionnaire for calcium and vitamin $D$ intake in adolescent girls with anorexia nervosa. J Am Diet Assoc. 2009;109:479-85.

11. Freedberg IM, Eisen AZ, Wolff K, et al., editores. Fitzpatrick's Dermatology in general medicine. 6th ed. New York McGraw-Hill; 2003.

12. Holick MF, Binkley NC, Bischoff-Ferrari HA, et al. Evaluation, treatment, and prevention of vitamin $\mathrm{D}$ deficiency an Endocrine Society Clinical Practice Guideline. J Clin Endocrinol Metab. 2011;96:1911-30.

13. Abbasian $M$, Chaman $R$, Amiri $M$, et al. Vitamin $D$ deficiency in pregnant women and their neonates. Glob J Healt Sci. 2016;8:83-90.

14. López-González D, Méndez-Sánchez L, Guagnelli MA, et al. Deficiencia de vitamina $D$ en la edad pediátrica. Una oportunidad de prevención. Boletín Médico del Hospital Infantil de México. 2015;72:225-34.

15. Sánchez Muro JM, Yeste Fernández D, Marín Muñoz A, et al. Niveles plasmáticos de vitamina $\mathrm{D}$ en población autóctona y en poblaciones inmigrantes de diferentes etnias menores de 6 años de edad. An Pediatr (Barc). 2015;82:316-24.

16. Sánchez $A$. Vitamina $D$ en el embarazo: su importancia para la madre y el feto. Actual Osteol. 2014;10:265-9.

17. Dror DK, King JC, Fung EB, et al. Evidence of associations between feto-maternal vitamin $\mathrm{D}$ status, cord parathyroid hormone and bone-specific alkaline phosphatase, and newborn whole body bone mineral content. Nutrients. 2012;4:68-77.

18. Marshall I, Mehta R, Petrova A. Vitamin D in the maternal-fetal-neonatal interface clinical implications and requirements for supplementation. J Matern Fetal Neonatal Med. 2013;26:633-8.

19. Dawodu A, Wagner CL. Mother-child vitamin D deficiency an international perspective. Arch Dis Child. 2007;92:737-40.

20. Sánchez A. Vitamina D actualización. Rev Med Rosario. 2010;76:70-82.

21. Flores M, Sánchez-Romero LM, Macías N, et al. Concentraciones séricas de vitamina $D$ en niños, adolescentes y adultos mexicanos. Resultados de la ENSANUT 2006. México Instituto Nacional de Salud Pública; 2006. p. $15-26$.

22. Elizondo-Montemayor L, Ugalde-Casas PA, Serrano-González M, et al. Serum 25-hydroxyvitamin D concentration, life factors and obesity in Mexican children. Obesity. 2010;18:1805-11.

23. Chan GM, McElligott K, McNaught $\mathrm{T}$, et al. Effects of dietary calcium intervention on adolescent mothers and newborns. Obstet Gynecol. 2006;108:565-71.

24. Diogenes ME, Bezerra FF, Rezende EP, et al. Effect of calcium plus vitamin $D$ supplementation during pregnancy in Brazilian adolescent mothers a randomized, placebo-controlled trial. Am J Clin Nutr. 2013;98:82-91.

25. Young BE, McNanley TJ, Cooper EM, et al. Vitamin D insufficiency is prevalent and vitamin $D$ is inversely associated with parathyroid hormone and calcitriol in pregnant adolescents. J Bone Miner Res. 2012; 27:177-86.

26. Soares MJ, Chan She Ping-Delfos W, Ghanbari MH. Calcium and vitamin $\mathrm{D}$ for obesity a review of randomized controlled trials. Eur $\mathrm{J}$ Clin Nutr. 2011;65:994-1004.

27. Awad $A B$, Alappat $L$, Valerio M. Vitamin $D$ and metabolic syndrome risk factors evidence and mechanisms. Crit Rev Food Sci Nutr. 2012; 52:103-12.

28. Masvidal Aliberch RM, Ortigosa Gómez S, Baraza Mendoza MC, et al Vitamina D: fisiopatología y aplicabilidad clínica en pediatría. An Pedia$\operatorname{tr}$ (Barc). 2012;77:279.e1-10.

29. Loya-López GM, Godínez-Gutiérrez SA, Chiquete E, et al. Niveles de vitamina $D$ en pacientes con sobrepeso y obesidad y su asociación con resistencia a la insulina. Revista de Endocrinología y Nutrición. 2011;19:140-5.

30. Misra M, Pacaud D, Petryk A, et al. Vitamin D deficiency in children and its management review of current knowledge and recommendations. Pediatrics. 2008;122:398.

31. Holick MF. Sunlight and vitamin D for bone health and prevention of autoimmune diseases, cancers, and cardiovascular disease. Am J Clin Nutr. 2004;80(6 Suppl):1678S-88S.

32. Togo A, Espadas M, Blanes Segura S, et al. ¿Existe déficit de vitamina $\mathrm{D}$ en los niños de una ciudad soleada del Mediterráneo? An Pediatr (Barc). 2016;84:163-9.

33. Bonilla C, Ness AR, Wills AK, et al. Skin pigmentation, sun exposure and vitamin $D$ levels in children of the Avon Longitudinal Study of Parents and Children. BMC Public Health. 2014;14:597.

34. Bodnar LM, Simhan HN, Powers RW, et al. High prevalence of vitamin D insufficiency in black and white pregnant women residing in the northern United States and their neonates. J Nutr. 2007;137:447-52.

35. Karras S, Paschou SA, Kandaraki E, et al. Hypovitaminosis D in pregnancy in the Mediterranean region a systematic review. Eur J Clin Nutr. 2016;70:979-86.

36. Ayadia ID, Nouailia EB, Talbi E, et al. Prevalence of vitamin D deficiency in mothers and their newborns in a Tunisian population. Int J Gynaecol Obstet. 2016;133:192-5.

37. Instituto Nacional de Estadística, Geografía e Informática (INEGI). Prontuario de información geográfica municipal de los Estados Unidos Mexicanos. Monterrey, Nuevo León. Disponible en http//www3.inegi.org.mx/ sistemas/mexicocifras/datos-geograficos/19/19039.pdf

38. Urrutia-Pereira $M$, Solé $D$. Vitamin $D$ deficiency in pregnancy and its impact on the fetus, the newborn and in childhood. Rev Paul Pediatr. 2015;33:104-13.

39. Ortigosa Gómez S, García-Algar O, Mur Sierra A, et al. Concentraciones plasmáticas de $25-\mathrm{OH}$ vitamina $\mathrm{D}$ y parathormona en sangre de cordón umbilical. Rev Esp Salud Pública. 2015;89:75-83.

40. Aghajafari F, Field CJ, Kaplan BJ, et al. The current recommended Vitamin $D$ intake guideline for diet and supplements during pregnancy is not adequate to achieve vitamin $\mathrm{D}$ sufficiency for most pregnant women. PLoS One. 2016;11:e0157262.

41. Wagner CL, Greer FR. American Academy of Pediatrics Section on Breastfeeding; American Academy of Pediatrics Committee on Nutrition. Prevention of rickets and vitamin D deficiency in infants, children, and adolescents. Pediatrics. 2008;122:1142-52. 